\title{
Human PTRF mutations cause secondary deficiency of caveolins resulting in muscular dystrophy with generalized lipodystrophy
}

\author{
Yukiko K. Hayashi, ${ }^{1}$ Chie Matsuda, ${ }^{2}$ Megumu Ogawa, ${ }^{1}$ Kanako Goto, ${ }^{1}$ Kayo Tominaga, ${ }^{1}$ \\ Satomi Mitsuhashi, ${ }^{1}$ Young-Eun Park, ${ }^{1}$ Ikuya Nonaka, ${ }^{1}$ Naomi Hino-Fukuyo, ${ }^{3}$ Kazuhiro Haginoya, ${ }^{3,4}$ \\ Hisashi Sugano, ${ }^{5}$ and Ichizo Nishino ${ }^{1}$

\begin{abstract}
1Department of Neuromuscular Research, National Institute of Neuroscience, National Center of Neurology and Psychiatry, Kodaira, Tokyo, Japan. ${ }^{2}$ Neuroscience Research Institute, National Institute of Advanced Industrial Science and Technology, Tsukuba, Ibaraki, Japan. ${ }^{3}$ Department of Pediatrics, Tohoku University School of Medicine, Sendai, Miyagi, Japan. ${ }^{4}$ Department of Pediatric Neurology, Takuto Rehabilitation Center for Children, Sendai, Miyagi, Japan. ${ }^{5}$ Department of Metabolic and Endocrine Medicine, Kochi Health Science Center, Kochi, Kochi, Japan.
\end{abstract}

\begin{abstract}
Caveolae are invaginations of the plasma membrane involved in many cellular processes, including clathrinindependent endocytosis, cholesterol transport, and signal transduction. They are characterized by the presence of caveolin proteins. Mutations that cause deficiency in caveolin-3, which is expressed exclusively in skeletal and cardiac muscle, have been linked to muscular dystrophy. Polymerase I and transcript release factor (PTRF; also known as cavin) is a caveolar-associated protein suggested to play an essential role in the formation of caveolae and the stabilization of caveolins. Here, we identified PTRF mutations in 5 nonconsanguineous patients who presented with both generalized lipodystrophy and muscular dystrophy. Muscle hypertrophy, muscle mounding, mild metabolic complications, and elevated serum creatine kinase levels were observed in these patients. Skeletal muscle biopsies revealed chronic dystrophic changes, deficiency and mislocalization of all 3 caveolin family members, and reduction of caveolae structure. We generated expression constructs recapitulating the human mutations; upon overexpression in myoblasts, these mutations resulted in PTRF mislocalization and disrupted physical interaction with caveolins. Our data confirm that PTRF is essential for formation of caveolae and proper localization of caveolins in human cells and suggest that clinical features observed in the patients with PTRF mutations are associated with a secondary deficiency of caveolins.
\end{abstract}

\section{Introduction}

Caveolae are specific invaginations of the plasma membrane characterized by the presence of the protein caveolin. To date, 3 caveolin family members have been identified. Caveolin- 1 and -2 are coexpressed in many cell types, such as endothelial cells, smooth muscle cells, fibroblasts, and adipocytes, and form a hetero-oligomeric complex (1). In contrast, caveolin-3 is expressed exclusively in skeletal and cardiac muscles (2). Caveolae are involved in several important cellular processes, including clathrin-independent endocytosis, regulation and transport of cellular cholesterol, and signal transduction $(3,4)$.

Polymerase I and transcript release factor (PTRF; also known as cavin) is a highly abundant caveolae component and is suggested to have an essential role in caveolar formation. In both mammalian cells and zebrafish, knockdown of PTRF leads to a reduction in caveolae density (5). Mice lacking PTRF do not have morphologically detectable caveolae, in addition to a markedly diminished protein expression of all 3 caveolin isoforms (6). Interestingly, PTRF-knockout mice mimic lipodystrophy in humans, demonstrating considerably reduced adipose tissue mass, high circulating triglyceride levels, glucose intolerance, and hyperinsulinemia (6).

Here we report that mutations in PTRF (GenBank accession no. 284119) caused a disorder presenting as generalized lipodystrophy and muscular dystrophy. We demonstrate that this condition was associated with deficiency and mislocalization of all 3 caveolin family members and reduction of caveolae structure.

Conflict of interest: The authors have declared that no conflict of interest exists. Citation for this article: J. Clin. Invest. 119:2623-2633 (2009). doi:10.1172/JCI38660.

\section{Results}

Identification of PTRF mutations. Deficiency of caveolin-3 as a result of CAV3 gene mutations is known to cause muscular dystrophy (7). We found 5 nonconsanguineous Japanese patients whose muscle showed caveolin-3 deficiency but without CAV3 mutation among 2,745 muscular dystrophy specimens kept in the muscle repository of the National Center of Neurology and Psychiatry. Importantly, all 5 patients also had congenital generalized lipodystrophy (CGL; also known as Berardinelli-Seip syndrome). From the findings observed in lacking cells and animal models lacking PTRF $(5,6)$, we screened for PTRF mutations.

We identified 2 different frameshift mutations in all 5 patients examined: patients 1-4 (P1-P4) had the same homozygous c.696_697insC (p.K233fs) mutation in exon 2, and P5 harbored a compound heterozygous mutation of the same c.696_697insC and c.525delG (p.E176fs) in exon 2 (Figure 1A). The c.525delG mutation changes the last 275 amino acids to an unrelated 98-amino acid sequence, whereas c.696_697insC substitutes the last 158 amino acids with an unrelated 191-amino acid sequence (Figure 1B). Both mutations were not identified in the chromosomes of 200 Japanese control subjects.

In order to determine whether the common c.696_697insC mutation has the same haplotype, we examined 6 sets of single nucleotide polymorphisms (SNPs) within PTRF: rs2062213, rs8070945, rs963988, rs963987, rs963986, and rs9252. All 5 patients had the same haplotype for all 6 SNPs, which occurred homozygously (Table 1). During mutation screening, we found a novel 9-bp insertion polymorphism in the $3^{\prime}$ noncoding region 


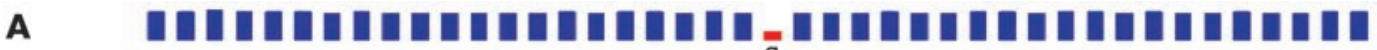

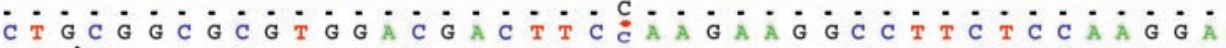
Con

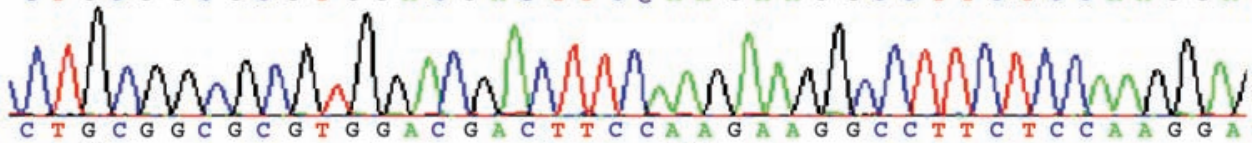

P1 InsC

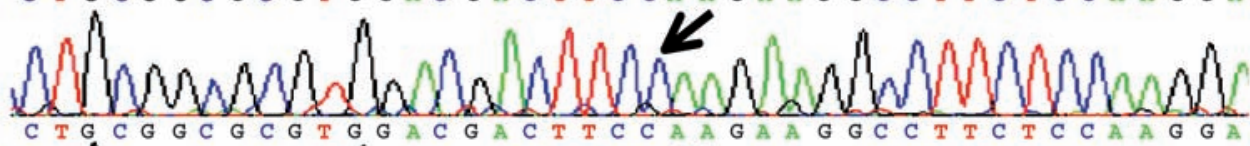
(homo)

P2 InsC

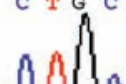
ก

(homo)

P3 InsC (homo)
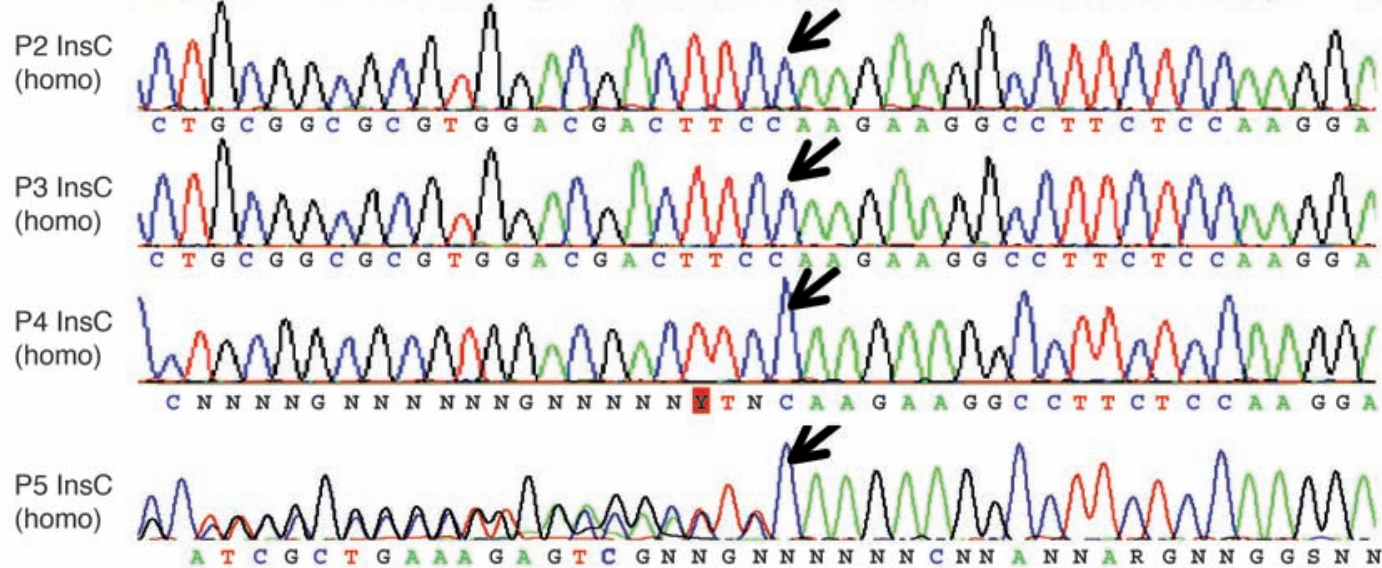

DelG

(hetero) A T C G C T G A A G A G C GN N G N N N N N C N A N N A R G N G G S N

B

B WT full length

1

DelG $175+98$ aa

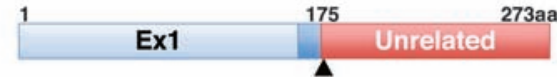

InsC 232+191 aa

$\begin{array}{ccc}1 & & 232 \\ \begin{array}{c}\text { PTRF } \\ \text { Antibodies }\end{array} & \begin{array}{c}\text { A301-269A } \\ \text { aa } 125-175\end{array} & \begin{array}{c}\text { A301-271A } \\ 238-288\end{array}\end{array}$

Figure 1

Mutations in PTRF. (A) All 5 patients had a homozygous or compound heterozygous mutation in PTRF (shown by arrows). P1-P4 had the same homozygous insertion mutation of c.696_697insC (InsC) in exon 2, whereas P5 had a compound heterozygous mutation of the same c.696_697insC insertion mutation and a deletion mutation of c.525delG (DelG) in exon 2. (B) Schema of the position of mutations in PTRF, putative proteins produced by mutations, and antibody recognition sites. The c.525delG mutant changes the last 275 amino acids to an unrelated 98-amino acid sequence, while the c.696_697insC mutant substitutes the last 158 amino acids with an unrelated 191-amino acid sequence.

of PTRF (c.1235_1236insTCTCGGCTC). This 9-bp insertion was found heterozygously in $26 \%$ and homozygously in $2 \%$ of Japanese control individuals. In P1-P5, none had this 9-bp insertion. We also examined 2 microsatellite markers (STS-W93348 and D17S1185) close to PTRF and found heterozygosity in the patients (Table 1). From these results, a founder effect may not be likely, although we could not completely rule out the possibility.

Mutation screening of the other genes associated with lipodystrophy and muscular dystrophy. From the clinical and pathological findings, we performed mutation screening for the genes associated with muscular dystrophy and lipodystrophy, including CAV3, LMNA, AGPAT2, BSCL2, CAV1, PPARG, AKT2, and ZMPSTE24. We found a heterozygous nucleotide change of c.1138G>A (p.D380N) in BSCL2 in P1. This substitution was also identified heterozygously in $16 \%$ of Japanese control individuals, and we believe this to be a novel nonsynonymous SNP. For all the other genes examined, no other mutation was identified in P1-P5.

Clinical features of the patients with PTRF mutations. Clinical information for P1-P5 is summarized in Table 2. Common to all patients was the presence of muscular dystrophy and generalized lipodystrophy. However, despite having the same mutation, the patients' additional symptoms were variable. Generalized loss of subcutaneous adipose tissue in several areas, including the face, was noticed in infancy or early childhood. Hepatosplenomegaly, acromegaloid features, and umbilical prominence were often observed in the patients. No patient showed intellectual deficit or acanthosis nigricans. Patients presented with mild muscle weakness, but with hypertrophy of muscles (Figure 2A). Electrically silent percussion-induced 
Table 1

Haplotype analysis

\begin{tabular}{lcccccc}
\hline & & & & & & \\
Intron 1, rs2062213 & $\mathrm{C} / \mathrm{C}$ & $\mathrm{C} / \mathrm{C}$ & $\mathrm{C} / \mathrm{C}$ & $\mathrm{C} / \mathrm{C}$ & $\mathrm{C} / \mathrm{C}$ & $\mathrm{C} / \mathrm{C}(53 \%)$ \\
Intron 1, rs8070945 & $\mathrm{C} / \mathrm{C}$ & $\mathrm{C} / \mathrm{C}$ & $\mathrm{C} / \mathrm{C}$ & $\mathrm{C} / \mathrm{C}$ & $\mathrm{C} / \mathrm{C}$ & $\mathrm{C} / \mathrm{C}(78 \%)$ \\
Intron 1, rs963988 & $\mathrm{G} / \mathrm{G}$ & $\mathrm{G} / \mathrm{G}$ & $\mathrm{G} / \mathrm{G}$ & $\mathrm{G} / \mathrm{G}$ & $\mathrm{G} / \mathrm{G}$ & $\mathrm{G} / \mathrm{G}(33 \%)$ \\
Intron 1, rs963987 & $\mathrm{G} / \mathrm{G}$ & $\mathrm{G} / \mathrm{G}$ & $\mathrm{G} / \mathrm{G}$ & $\mathrm{G} / \mathrm{G}$ & $\mathrm{G} / \mathrm{G}$ & $\mathrm{G} / \mathrm{G}(31 \%)$ \\
Intron 1, rs963986 & $\mathrm{G} / \mathrm{G}$ & $\mathrm{G} / \mathrm{G}$ & $\mathrm{G} / \mathrm{G}$ & $\mathrm{G} / \mathrm{G}$ & $\mathrm{G} / \mathrm{G}$ & $\mathrm{G} / \mathrm{G}(34 \%)$ \\
Exon 2, 9-bp insertion & no/no & no/no & no/no & no/no & no/no & no/no $(72 \%)$ \\
Exon 2, rs9252B & $\mathrm{C} / \mathrm{C}$ & $\mathrm{C} / \mathrm{C}$ & $\mathrm{C} / \mathrm{C}$ & $\mathrm{C} / \mathrm{C}$ & $\mathrm{C} / \mathrm{C}$ & $\mathrm{C} / \mathrm{C}(78 \%)$ \\
STS-W93348 (bp) & $251 / 253$ & $251 / 253$ & $251 / 253$ & $251 / 253$ & $251 / 253$ & $251 / 253 / 264$ \\
D17S1185 (bp) & $219 / 219$ & $170 / 219$ & $170 / 219$ & $170 / 170$ & $170 / 203$ & $170 / 203 / 215 / 219 / 225 / 237$
\end{tabular}

APercentages denote the frequency of the haplotype in the HapMap JPT population. ${ }^{\mathrm{B} E x o n} 2$ is $3^{\prime}$ noncoding.

analysis revealed a heterozygous c.696_698insC mutation in both parents of P4. Clinically, both father and mother had hypertension requiring medication, whereas P4 was normotensive. Mild lipid metabolism abnormality and borderline glucose intolerance was also seen (Supplemental Table 2). DNA samples from the other parents were not available.

Loss of PTRF with deficiency or mislocalization of caveolins in skeletal muscle. Biopsied skeletal muscles from P1-P5 showed consistent findings, with chronic dystrophic changes

muscle mounding was characteristic. Cardiac arrhythmia, transient immunodeficiency, recurrent pneumonia, constipation, and chalasia were variably seen. Available laboratory data in the patients are summarized in Table 3. Metabolic complication was mild, and none of our patients showed marked elevation of fasting glucose levels. The result of oral glucose tolerance tests revealed moderate fasting hyperinsulinemia in P1 and P2 associated with glucose intolerance in P2, but normal levels in P4 (Table 4). High triglyceremia was seen in P4 and P5. Serum creatine kinase (CK) levels were moderately elevated in all patients. Abdominal CT images of $\mathrm{P} 4$ revealed marked loss of subcutaneous and intra-abdominal fat (Figure 2, B and C). In addition, his body fat ratio, as determined by whole body dual energy X-ray absorptiometry, was 7.1\% (Supplemental Table 1; supplemental material available online with this article; doi:10.1172/ JCI38660DS1), while head fat was relatively preserved.

Clinical features of the heterozygous parents. There was no family history of muscular dystrophy or lipodystrophy in P1-P5. Genetic including marked variation in muscle fiber size, increased number of fibers containing internalized nuclei, a few necrotic and regenerating fibers, and increased interstitial fibrosis (Figure 2D and Supplemental Figure 1). Intramuscular lipid droplets, as visualized by oil red O staining, were not increased (Figure 2D).

Immunohistochemistry demonstrated that the PTRF antibodies A301-269A and A301-271A (which recognize the N- and C-terminal regions of the protein, respectively; Figure 1B) showed sarcolemmal membrane staining of muscle fibers, with stronger immunoreaction at intramuscular blood vessels in control muscles (Figure 3A). Caveolin-3 was clearly observed at sarcolemma, whereas caveolin-1 and - 2 were present only in blood vessels. In contrast, muscles from P1-P5 showed barely detectable immunoreaction to both PTRF antibodies (Figure 3A). Caveolin-3 immunoreactivity was greatly reduced in the sarcolemma, but cytoplasmic staining was remarkably increased. This caveolin-3 staining pattern was similar to that seen in the patients with muscular dystrophy

\section{Table 2}

Clinical summary

\begin{tabular}{|c|c|c|c|c|c|}
\hline & P1 & $\mathbf{P} 2$ & P3 & P4 & P5 \\
\hline Age/sex & 8-yr-old female & 14-yr-old female & 10-yr-old male & 27-yr-old male & 24-yr-old male \\
\hline Height, body weight & $124 \mathrm{~cm}, 21.3 \mathrm{~kg}$ & $149 \mathrm{~cm}, 40.5 \mathrm{~kg}$ & NA & $164 \mathrm{~cm}, 49.0 \mathrm{~kg}$ & $152 \mathrm{~cm}, 40 \mathrm{~kg}$ \\
\hline Lipodystrophy & Generalized & Generalized & Generalized & Generalized & Generalized \\
\hline Mental retardation & No & No & No & No & No \\
\hline Acanthosis nigricans & No & No & No & No & No \\
\hline Liver/spleen & Hepatosplenomegaly & Fatty liver & NA & Hepatosplenomegaly & No \\
\hline Endocrine abnormalities & $\begin{array}{l}\text { Reduced growth } \\
\text { hormone secretion }\end{array}$ & NA & NA & $\begin{array}{l}\text { Accelerated bone age, } \\
\text { acromegaloid features, } \\
\text { no androgynism }\end{array}$ & $\begin{array}{l}\text { Acromegaloid features, } \\
\text { no androgynism }\end{array}$ \\
\hline Muscle weakness & Distal dominant & No & No & Generalized & Distal dominant \\
\hline Muscle mounding & Positive & NA & NA & Positive & Positive \\
\hline Other muscle symptoms & Muscle hypertrophy & $\begin{array}{l}\text { Myalgia, } \\
\text { muscle stiffness }\end{array}$ & NA & Muscle hypertrophy & Muscle hypertrophy \\
\hline Cardiac symptoms & Arrhythmia & No & No & Atrial fibrillation & No \\
\hline Skeletal abnormalities & $\begin{array}{l}\text { Lordosis, } \\
\text { Contractures (ankles, } \\
\text { shoulders, fingers) }\end{array}$ & No & No & $\begin{array}{l}\text { Scoliosis, } \\
\text { contractures (ankles) }\end{array}$ & Scoliosis \\
\hline Other symptoms & Constipation & $\begin{array}{l}\text { Transient IgA deficiency, } \\
\text { recurrent pneumonia }\end{array}$ & Nephrosis & $\begin{array}{l}\text { Umbilical prominence, } \\
\text { renal stones }\end{array}$ & $\begin{array}{l}\text { Recurrent pneumonia, } \\
\text { chalasia, constipation }\end{array}$ \\
\hline
\end{tabular}

NA, not available. 

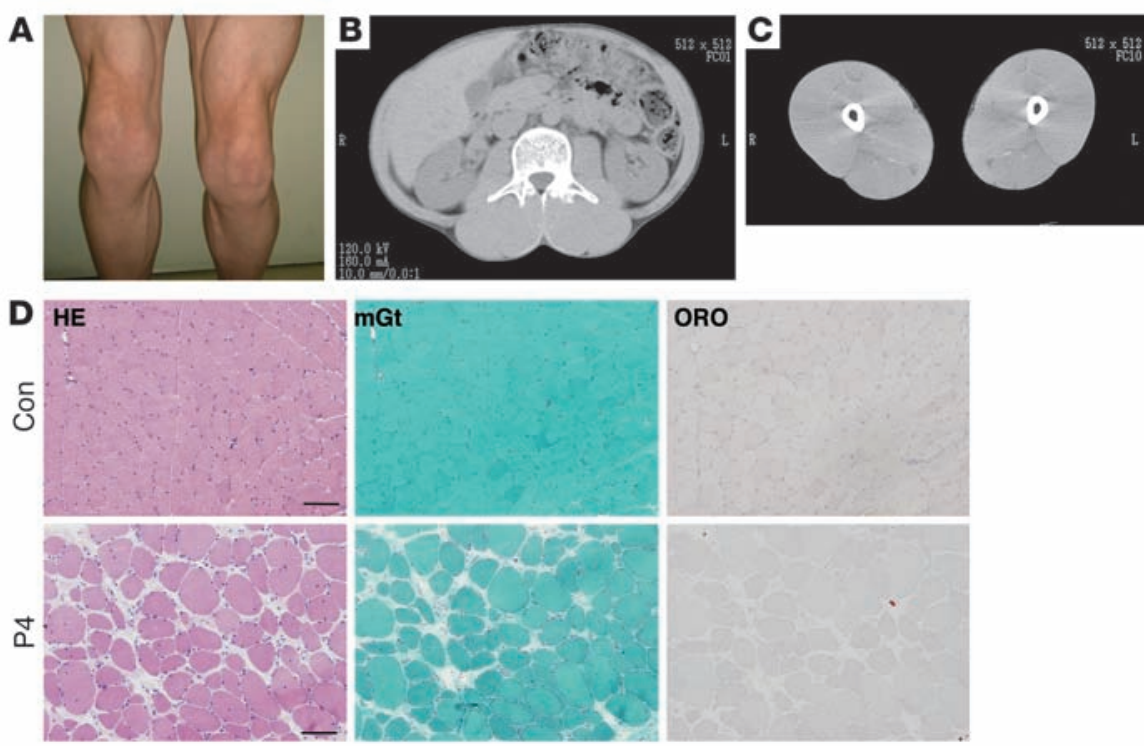

\section{Figure 2}

Muscle hypertrophy and dystrophic changes. (A) Prominent musculature feature of legs in P5. (B and C) CT images from P4 showed hypertrophy of paravertebral and thigh muscles with minimal subcutaneous and intra-abdominal fat tissue. (D) H\&E stain of biopsied skeletal muscle from P4 showed dystrophic changes, including marked variation in fiber size, enlarged fibers with internalized nuclei, endomysial fibrosis, and few necrotic and regenerating fibers. Intramuscular lipid droplets were not increased compared with control. mGt, modified Gomori trichrome; ORO, oil red O. Scale bars: $50 \mu \mathrm{m}$. caused by CAV3 mutations (data not shown). Similarly, dysferlin was decreased in the sarcolemma and mislocalized into the cytoplasm (data not shown), and the same pattern is also seen in muscles of individuals with CAV3 mutations (8). Immunoreactivity to caveolin-1 and caveolin-2 in blood vessels was barely detectable in P1-P5 (Figure 3A). Other antibodies related to muscular dystrophy, including dystrophin, sarcoglycans, dystroglycans, emerin, merosin, and collagen VI, showed normal immunostaining patterns (data not shown).

Immunoblotting showed detection of PTRF as an approximately $50-\mathrm{kDa}$ band in control muscles and $3 \mathrm{~T} 3$ cells, which were used as a positive control. No band was detected in the muscle of P1-P5 (Figure 3B). Caveolin-3 was detected in all samples examined, but relative protein amount, determined using densitometry and normalized by myosin heavy chain (MHC), decreased in P1-P5 compared with control subjects (Figure $3 \mathrm{C}$ ). The band for caveolin-2 was observed in control muscles and $3 \mathrm{~T} 3$ cells, but was barely detectable in the muscles of P1-P5 (Figure 3B).

In order to determine mRNA expression of PTRF, RT-PCR was performed using total RNA extracted from biopsied skeletal muscles. Using primers designed to amplify whole coding region of mRNA, PTRF was amplified as a single transcript in control muscles. In contrast, no PCR product was amplified in P1-P5 (Figure 4A). To compare mRNA levels for caveolins, we performed quantitative RT-PCR and normalized results to GAPDH expression. The mRNA amounts of all 3 caveolin families in the patients' muscles were variable, but not markedly decreased, compared with control muscles (Figure 4, B and C). Preserved mRNA levels, but decreased protein amounts of caveolins, suggested destabilization of caveolin proteins when PTRF is lacking, as previously reported (9).

Loss of PTRF causes reduced caveolae formation in buman muscles. Greatly reduced caveolae formation was previously reported in PTRF knockdown mammalian cells, zebrafish, and knockout mice $(5,9)$. Decreased caveolae number was also reported in skeletal muscle from limb girdle muscular dystrophy type 1C (LGMD1C) patients with CAV3 mutations (10). We therefore examined muscle caveolae in P2 and P3 using electron microscopy. Plasma membrane of muscle fibers from both patients was nearly flat, and caveolae density was notably reduced, compared with control muscle (Figure 5). Caveolae formation in the intramuscular vascular smooth muscle cells was also remarkably reduced (data not shown).

Altered localization of mutant PTRF and reduced interaction with caveolins in transfected cells. In order to determine the intracellular localization of mutant PTRF, FLAG-tagged WT or 2 mutants (c.525delG and c.696_697insC) and T7-tagged caveolin-3 or -1 were cotransfected in C2C12 myoblasts and COS-7 cells. In C2C12 cells, WT PTRF was detected at the cell membrane and colocalized with caveolin-3 (Figure 6A). Interestingly, c.525delG was detected as intranuclear aggregations and was not observed at the cell membrane (Figure 6, A and B). Caveolin-3 was present only in cytoplasm, and did not merge with PTRF (Figure 6A). The c.696_697insC mutant was observed as microtubular filament network in cytoplasm and colocalized with $\beta$-tubulin (Figure 6B). This finding is consistent with the localization of the truncated $\mathrm{PTRF}_{1-322}$, as described previously (9). Similar mislocalization and/or aggregation of transfected mutant PTRF was observed in COS-7 cells (data not shown). 
Table 4

Oral glucose tolerance test of P1, P2, and P4

\begin{tabular}{lcccc} 
& Pre & $\mathbf{3 0} \mathbf{~ m i n}$ & $\mathbf{6 0} \mathbf{~ m i n}$ & $\mathbf{1 2 0} \mathbf{~ m i n}$ \\
P1 & & & & \\
Glucose $(\mathrm{mg} / \mathrm{dl})$ & 75 & 98 & 69 & 62 \\
IRI $(\mu \mathrm{U} / \mathrm{ml})$ & 22.8 & 141.6 & 64.7 & 23.8 \\
P2 & & & & \\
Glucose $(\mathrm{mg} / \mathrm{dl})$ & 99 & 127 & 160 & 172 \\
IRI $(\mu \mathrm{U} / \mathrm{ml})$ & 20 & 53 & 65 & 80 \\
P4 & & & & \\
Glucose $(\mathrm{mg} / \mathrm{dl})$ & 93 & 124 & 140 & 70 \\
CPR $(\mathrm{ng} / \mathrm{ml})^{\mathrm{A}}$ & 2.8 & 5.9 & 8.3 & 5.5 \\
IRI $(\mu \mathrm{U} / \mathrm{ml})$ & 1.0 & 22.3 & 32.9 & 6.2 \\
\hline
\end{tabular}

IRI, immunoreactive insulin; CPR, C-peptide immunoreactivity. AReference range, $0.7-2.2 \mathrm{ng} / \mathrm{ml}$.

We performed immunoprecipitation assay in order to examine the binding ability of PTRF and caveolins. WT PTRF was coimmunoprecipitated by anti-T7 antibody, and vice versa (Figure 6, C and D). The c.525delG mutant showed smaller molecular weight (estimated $30 \mathrm{kDa}$; Figure 1B), and no immunoprecipitated protein was detected by FLAG and T7 antibodies. The c.696_697insC mutant showed slightly larger molecular weight, and coimmunoprecipitated proteins were greatly reduced (Figure 6, C and D). These results suggest that mutant PTRFs cannot localize properly and lose their binding ability to caveolins even if they are produced.

Activation of myostatin and Akt signaling pathways in PTRF-deficient skeletal muscles. Caveolin-3 is suggested to have an important role for suppression of myostatin-mediated signaling in skeletal muscle (11). In order to determine the functions of mislocalized caveolin-3 in PTRF mutated cells, we performed quantitative RT-PCR for myostatin and immunoblotting analysis to examine phosphorylation status of Mad homolog 2/3 (p-Smad2/3), an intracellular effector of myostatin in skeletal muscles. In P1-P5, increased amounts of $\mathrm{p}-\mathrm{Smad} 2 / 3^{\mathrm{S} 423 / 425}$ were observed in skeletal muscles, while myostatin mRNA levels were variable (Figure $7, \mathrm{~A}-\mathrm{C}$ ). Positive immunoreaction to $\mathrm{p}-\mathrm{Smad} 2 / 3$ was detected in few myonuclei from muscle of patients with PTRF or CAV3 mutations, but not in those from muscle of control subjects (data not shown). These results suggest that myostatin signaling is also activated in P1-P5.

Despite the activation of myostatin, a negative regulator of muscle growth, the patients showed hypertrophy of muscles. Since Akt (also known as protein kinase B) is known as the key molecule to regulate muscle mass (12), we examined $\mathrm{p}-\mathrm{Akt}^{\mathrm{T} 308}$ and $\mathrm{p}-\mathrm{Akt}^{\mathrm{S} 473}$ by immunoblotting analysis. p-Akt was elevated in the muscle of $\mathrm{P} 1-\mathrm{P} 5$ compared with controls, except for $\mathrm{p}-\mathrm{Akt}^{\mathrm{S} 473}$ in $\mathrm{P} 2$ (Figure 7, $\mathrm{D}-\mathrm{F})$. This result suggests that Akt pathway is activated, probably through an as-yet-unidentified mechanism, and could contribute to the muscle hypertrophy observed in P1-P5.

Neuronal NOS activity is variable and mildly increased in PTRF-deficient skeletal muscles. Caveolin-3 is known to interact with and negatively regulate the catalytic activity of neuronal NOS (nNOS) in skeletal muscle (13); this notion is supported by the finding of increased nNOS activity in muscle of transgenic mice expressing mutant caveolin-3 (14). We thus examined nNOS expression and its activity in muscles from patients with mutations in PTRF or $C A V 3$ compared with those from age-matched controls. The immunoreactivity of nNOS was seen in sarcolemma and cytoplasm of each muscle fiber with variable intensity, but no obvious difference was seen between patients and controls (Figure 3A). Immunoblotting analysis also revealed comparable amounts of nNOS (Figure $3, \mathrm{~B}$ and D). In order to examine nNOS activity of each muscle fiber, we performed NADPH diaphorase (NDP) activity assay. The intensity of NDP staining appeared variable among muscle fibers and was slightly increased in patients with mutations in PTRF or CAV3 compared with age-matched controls (Figure 8).

\section{Discussion}

Lipodystrophy is a heterogeneous group of disorders characterized by loss of adipose tissue from the body. The degree of fat loss varies from small areas to near-complete absence of adipose tissue. The extent of fat loss usually determines clinical severity and metabolic complications, such as insulin resistance and high levels of serum triglycerides.

Several genes responsible for inherited lipodystrophy have been identified. CGL is an autosomal-recessive disorder, with most patients presenting soon after birth with severe insulin resistance and elevated serum triglycerides. CGL1 is caused by mutations in AGPAT2 on chromosome 9q34, which encodes 1-acylglycerol-3phospate-O-acyltransferase 2, an enzyme involved in the biosynthesis of triacylglycerol and glycerophospholipids (15). CGL2 is caused by mutations in BSCL2 on chromosome $11 \mathrm{q} 13$, which encodes a functionally unknown protein named seipin (16). Recently, mutations in CAV1 on chromosome $7 \mathrm{q} 31$ have been reported to cause generalized (i.e., CGL3) and partial lipodystrophy $(17,18)$.

Several causative genes for autosomal-dominant familial partial lipodystrophy are known: LMNA on chromosome 1q21 (19), ZMPSTE24 on chromosome 1p34 (20), AKT2 on chromosome 19q13 (21), PPARG on chromosome 3p25 (22), and LMNB2 on chromosome 19q1 (23). Nevertheless, many patients clinically diagnosed with lipodystrophy carry no mutation in the known genes, suggesting the presence of other causative genes.

Here we conclude that PTRF mutations can cause CGL. In our series, patients showed generalized loss of adipose tissue from infancy or early childhood. Because PTRF is reported to colocalize with hormone-sensitive lipase and translocate to the nucleus in the presence of insulin in adipocytes (24), it could be surmised that PTRF plays an important role in lipid metabolism and insulin-regulated gene expression. Interestingly, metabolic complications were milder in patients with PTRF mutations than in patients with CGL1 and CGL2, and these were observed only in the elder patients. Although we could not examine the status of caveolae and caveolins in adipose tissues, the secondary deficiency of caveolins might have an important role in the process of lipodystrophy, since CAV1 mutation can cause lipodystrophy in both humans and mice $(17,18,25)$. Notably, the heterozygous parents had mild metabolic disorders, but a robust conclusion could not be reached, as a limited number of the heterozygous carriers of the PTRF mutation were available to us. Further investigation is needed to determine the effect of haploinsufficiency of PTRF.

Skeletal muscle symptoms with serum CK elevation represent another common symptom in patients with PTRF mutations. The clinical and pathological findings are very similar to those observed in patients with CAV3 mutation (7, 26-28), although P1-P5 had no CAV3 mutations. The secondary loss of caveolin-3 in the sarcolemma may contribute to the muscle phenotype. Moreover, serum CK elevation may be a good laboratory marker for diagnosis of lipodystrophy patients with PTRF mutations. 
A
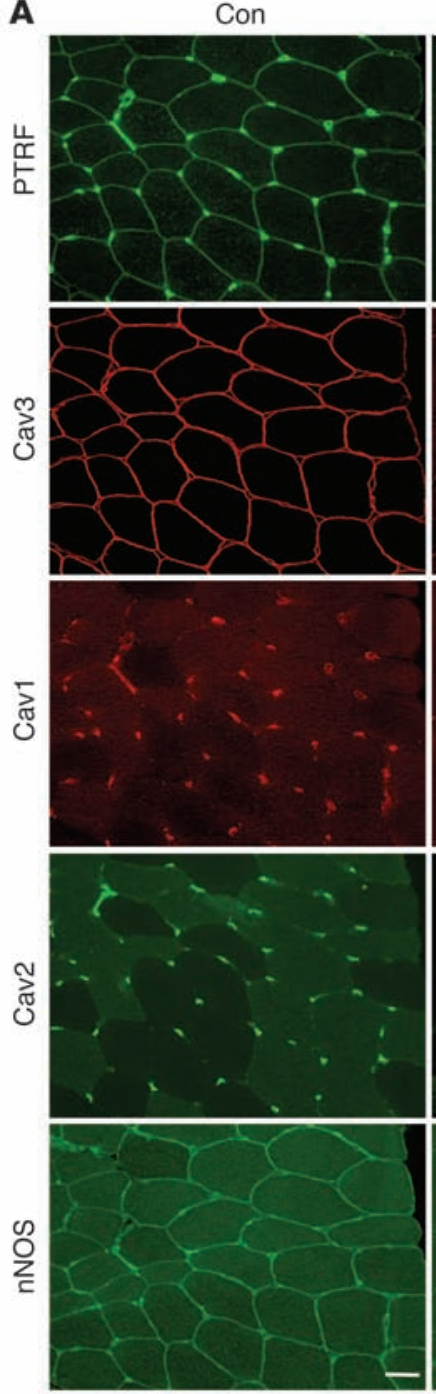

P4
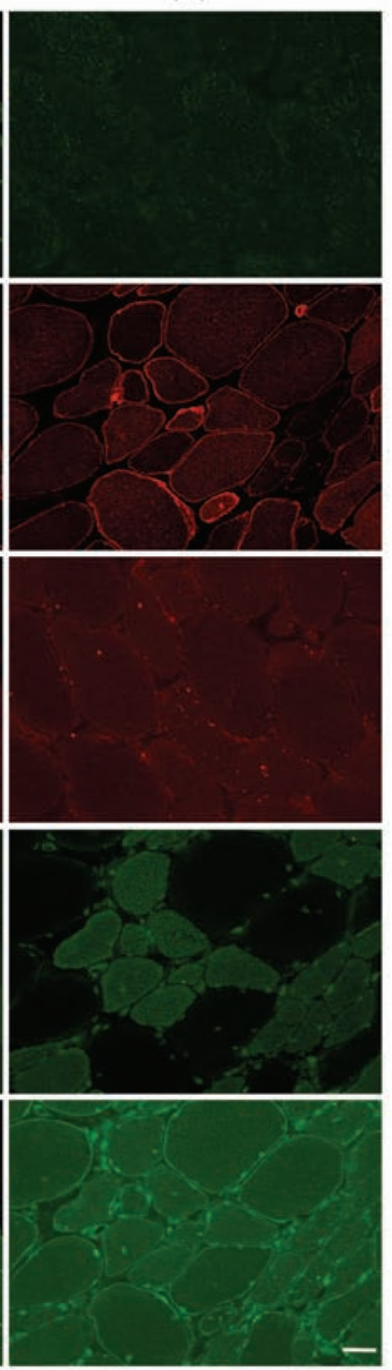

B

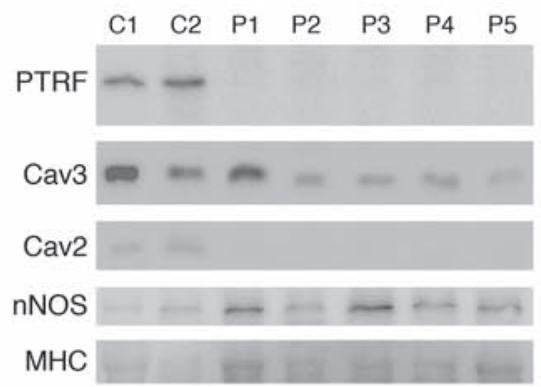

$\mathbf{C}_{7}$

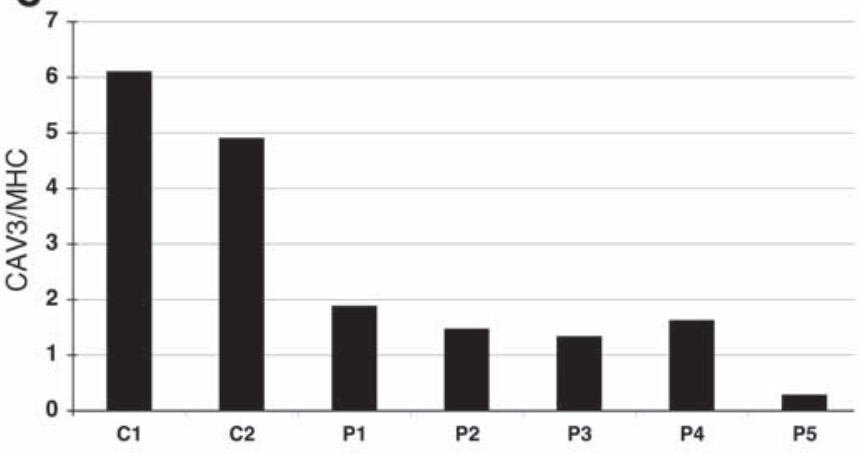

D

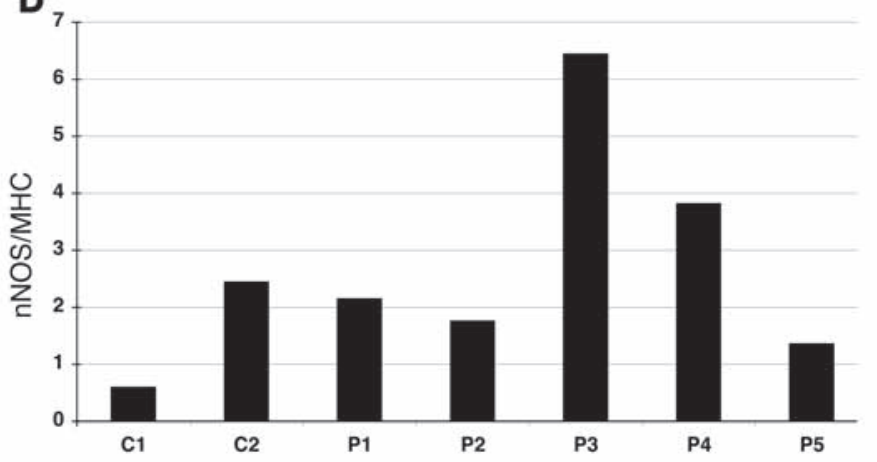

Figure 3

Loss of PTRF is associated with deficiency and mislocalization of caveolins in muscle. (A) In control muscle, PTRF was clearly seen in sarcolemma as strongly staining blood vessels. Caveolin-3 (Cav3) was clearly visible at sarcolemma, and caveion-1 and -2 stained intramuscular blood vessels. The muscle of P4 was negative for PTRF. Membrane staining of caveolin-3 was reduced with increased cytoplasmic staining, and caveolin-1 and -2 were barely detectable. Immunoreactivity of nNOS varied between muscle fibers, but was not markedly different between control and patient muscle. Scale bar: $50 \mu \mathrm{m}$. (B) Immunoblotting analysis of skeletal muscles. 3T3 cells were used as a positive control. PTRF and caveolin-2 were seen only in the muscles of 2 control subjects and in 3 T3 cells, and were barely detectable in the muscles of P1-P5. The bands for caveolin-3 and nNOS were variably seen. (C and D) Quantification of immunoreactive bands was performed by densitometric analysis and normalized with MHC. In P1-P5, relative amounts of caveolin-3 decreased compared with control subjects (C), whereas nNOS amounts varied (D).

Caveolin-3 was previously reported to have an important role in inhibition of myostatin signaling by suppressing activation of its type I receptor. In mutant Cav3 transgenic mice, loss of caveolin-3 causes muscular atrophy with increased $\mathrm{p}-\mathrm{Smad} 2$, and this muscle atrophy can be rescued by myostatin inhibition (11). Consistent with the secondary reduction of caveolin-3, skeletal muscles from P1-P5 showed increased amounts of p-Smad2/3. Unexpectedly, however, muscle hypertrophy was seen in these patients.

The Akt pathway, when activated, is known to promote protein synthesis, stimulate muscle hypertrophy, and inhibit atrophyrelated gene expression by phosphorylating FoxO transcription factors (12). This pathway is also known to play a pivotal role in the regulation of glucose transport and glycogen synthesis in skeletal muscle cells. Akt is activated by insulin, various growth factors, nutrients, and exercise, whereas it is negatively regulated by myostatin and cytokines. Akt is phosphorylated at T308 by phosphoinositide-dependent kinase and at S473 by mammalian target of rapamycin in association with rictor. The increase in phosphorylated Akt in the muscle of P1-P5 may explain, at least in part, the muscle hypertrophy observed. Akt pathway activation might be associated with the metabolic complications observed in P1-P5. However, the upregulation of myostatin observed is contradictory to the established knowledge on muscle hypertrophy. This would be worthwhile to investigate in future studies, in order to 
A
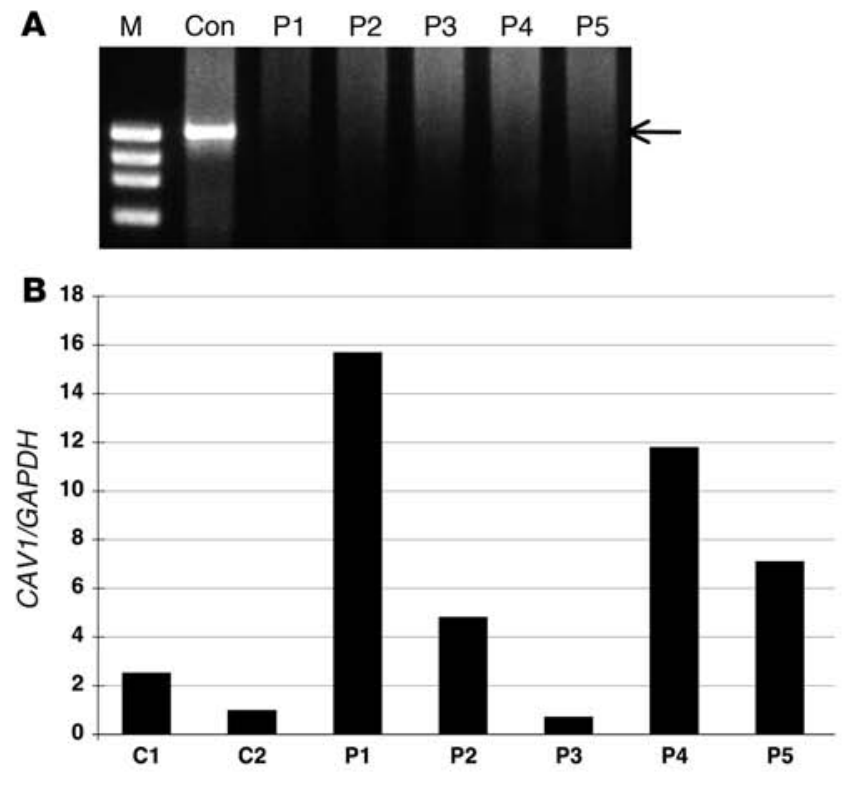

Figure 4

mRNA expression of PTRF in skeletal muscle, and quantitative RT-PCR of mRNAs for caveolins. (A) RT-PCR analysis revealed a single band for PTRF mRNA (arrow) in a control subject, but no detectable product was seen in P1-P5. M, marker. (B-D) By quantitative RT-PCR, mRNA for CAV1, CAV2, and CAV3 normalized with GAPDH expression was not decreased in P1-P5.

elucidate the role of PTRF deficiency in muscle hypertrophy and related signaling pathway.

In addition to lipodystrophy and muscular dystrophy, P1-P5 had various other symptoms, whose association to PTRF mutation might be difficult to ascertain at this time. For example, 2 of 5 patients had arrhythmia. Although we could not examine the expression of caveolins in cardiac muscle, this cardiac abnormality may be caused by secondary deficiency of caveolins in heart, as cardiac involvement was previously reported in patients with CAV3 mutations and in mutant mice with double knockout of Cav1 and Cav3 (29-33).

Remarkable reduction in expression of caveolin-1 and -2 with decreased caveolae density was observed in vascular endothelial cells in P1-P5. There was no obvious symptom related to vascular endothelial blood vessels in the patients; however, further careful investigation is necessary in order to determine the involvement of endothelial cells, which was observed in Cav1 knockout mice (34). The severe constipation and esophageal dilatation observed in the patients might be associated with dysfunction of caveolin-1 in smooth muscle cells, as Cav1 knockout mice had alteration of

\section{Figure 5}

Reduced caveolae formation in skeletal muscle, as assessed by electron microscopy. In control muscle, an abundance of caveolae (arrowheads) was observed close to the plasma membrane. Plasma membrane of muscle fibers from P2 and P3 was nearly flat, and caveolae density was greatly reduced compared with that of control muscle. Only a few caveolae were seen in P2. Scale bars: $200 \mathrm{~nm}$.
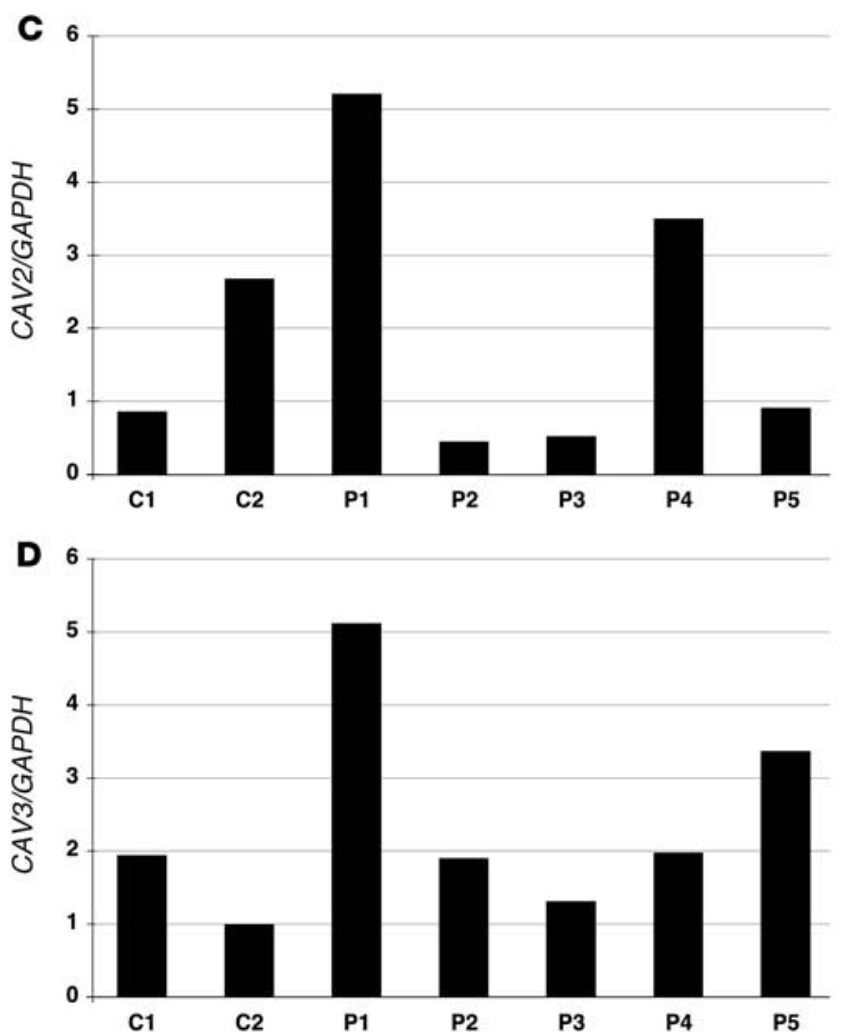
A PTRF (Anti-FLAG)

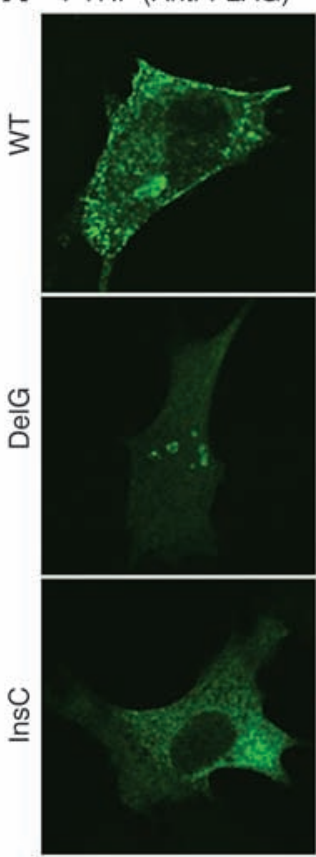

B PTRF (Anti-FLAG)
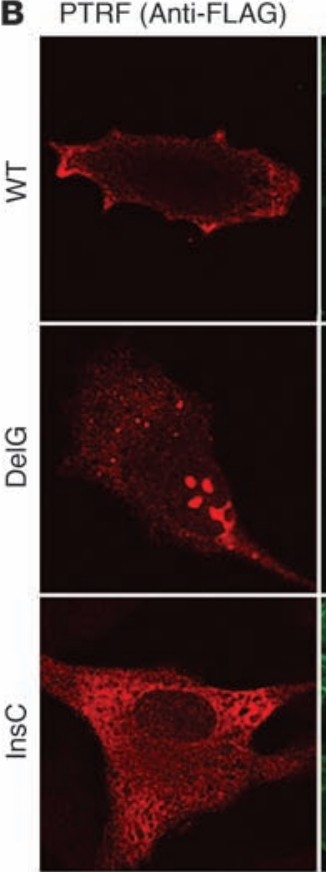

Cav3

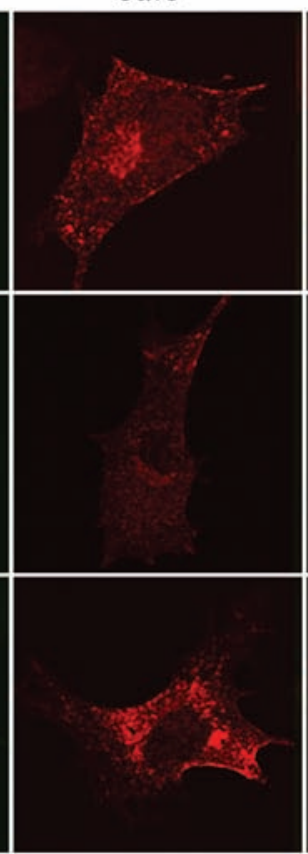

$\beta$-Tubulin

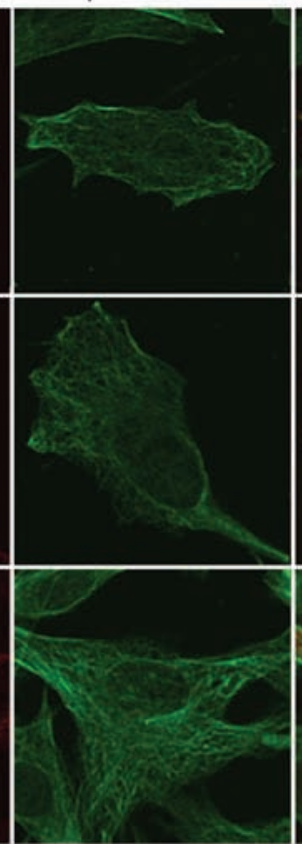

Merge

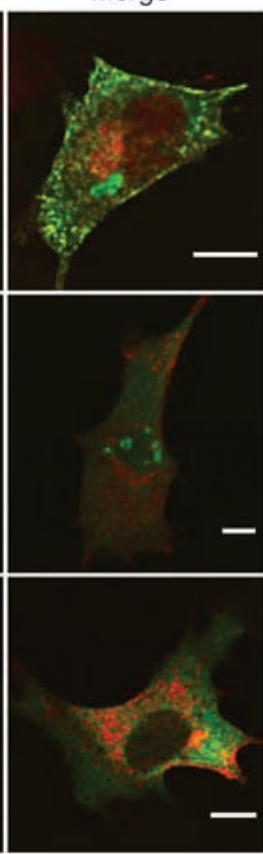

Merge

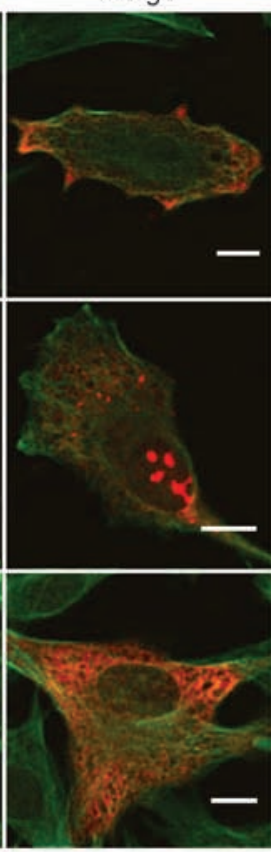

PTRF

(Anti-FLAG)
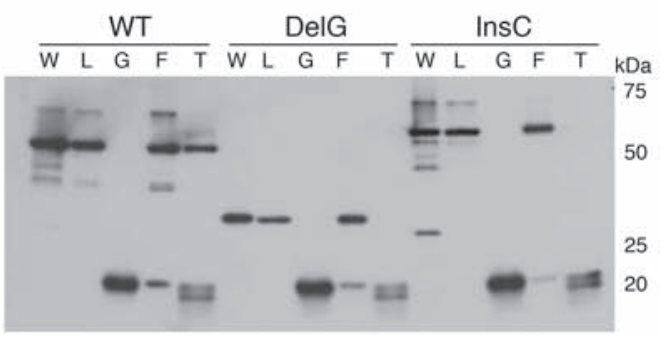

Cav3

(Anti-T7)

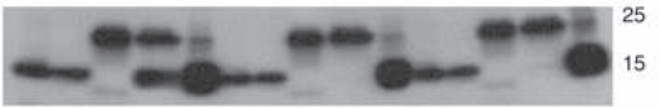

D

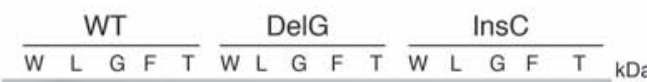

PTRF

(Anti-FLAG)

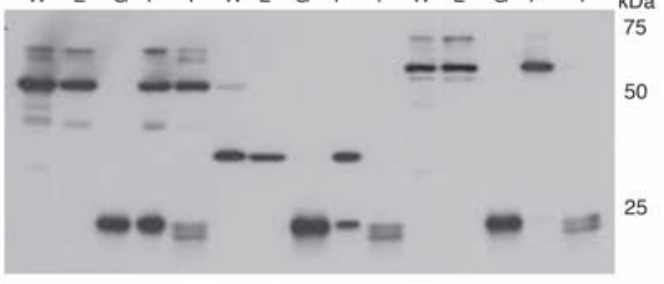

Cav1

(Anti-T7)

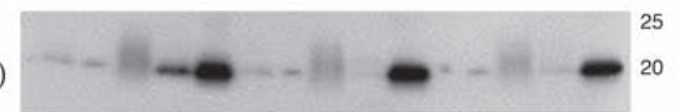

Figure 6

Altered localization of mutant PTRF in $\mathrm{C} 2 \mathrm{C} 12$ cells and reduced binding ability to caveolins. C2C12 myoblasts were cotransfected with FLAG-tagged WT or mutant (c.525delG or c.696_697insC) PTRF cDNA and T7-tagged human caveolin-3. (A and B) WT PTRF stained by anti-FLAG antibody colocalized with caveolin-3 at the cell membrane. The deletion mutant accumulated in the nucleus, and the insertion mutant was seen in cytoplasm. (A) Membrane staining of caveolin-3 was decreased and was not colocalized with mutant PTRF. (B) The PTRF insertion mutant clearly colocalized with $\beta$-tubulin. Scale bars: $10 \mu \mathrm{m}$. (C and D) COS-7 cells were cotransfected with FLAG-tagged WT or mutant PTRF cDNA and T7-tagged human caveolin-3 (C) or caveolin-1 (D). The PTRF deletion mutant showed smaller molecular weight (estimated $30 \mathrm{kDa}$ ), and no immunoprecipitated protein was detected for FLAG or T7 antibodies. The PTRF insertion mutant showed slightly larger molecular weight, and amounts of coimmunoprecipitated proteins were greatly reduced. W, whole homogenate; L, cell lysate, G, control IgG; F, anti-FLAG; T, anti-T7. 
A
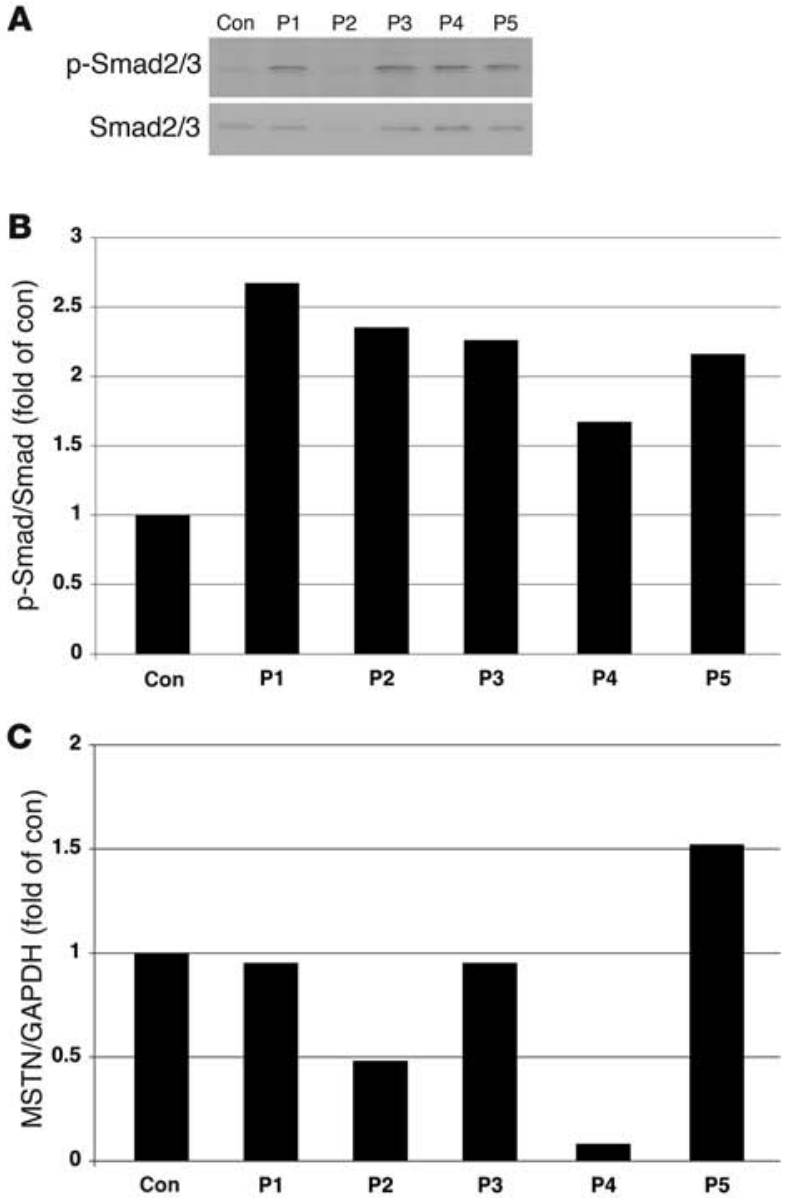

D

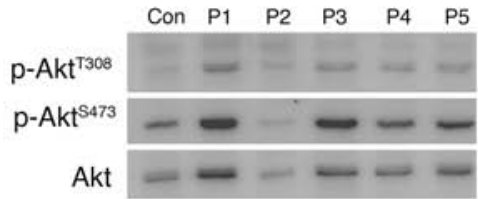

E
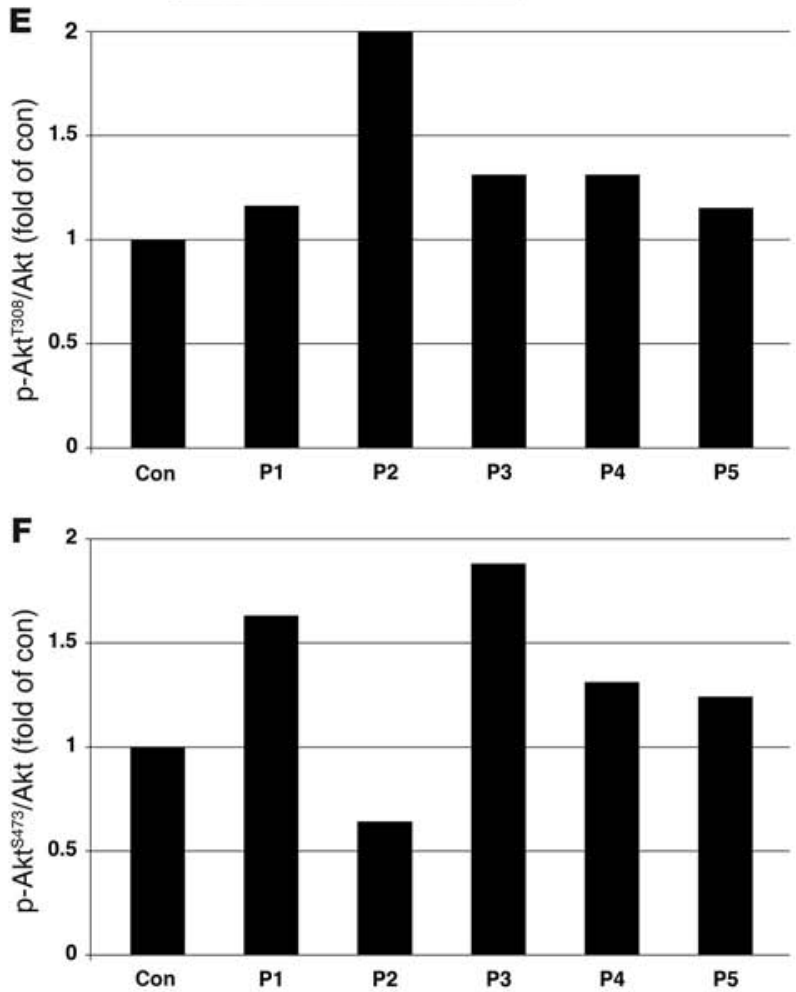

Figure 7

Increased p-Smad2 and p-Akt in P1-P5 skeletal muscle. (A-C) Immunoblotting analysis of Smad2/3 and p-Smad2/3S423/425 (A) and densitometric analysis (B) showed increased p-Smad2/3 in P1-P5 compared with control muscle, with variable mRNA expression levels of myostatin (MSTN; C). (D-F) Immunoblotting analysis of $\mathrm{p}-\mathrm{Akt}^{\mathrm{T} 308}$ and $\mathrm{p}-\mathrm{Akt}^{\mathrm{S}}{ }^{\mathrm{T} 3}$. Total $\mathrm{Akt}(\mathbf{D})$ and densitometric analysis (E and $\mathbf{F}$ ) showed increased amounts of $\mathrm{p}$-Akt in all patients except for $\mathrm{p}-\mathrm{Akt}^{\mathrm{S4}}{ }^{3}$ in $\mathrm{P} 2$.

most clinical features observed in P1-P5 are likely to be explained by secondary reduction of caveolae and deficiency of caveolins.

Previously, Rajab et al. reported 10 of 17 patients with congenital generalized lipodystrophy unlinked to the loci of known CGL genes (37). The patients showed reduced exercise tolerance, percussion myoedema, cardiac hypertrophy, and arrhythmias. None of these patients had insulin resistance or early endocrine abnormalities (37). Ghanem also reported myoedema in a patient with Berardinelli-Seip lipodystrophy (38). Very recently, Simha et al. described CGL patients with muscle weakness and cervical spine instability (39). Because muscle involvement of these patients is similar to that of P1-P5 in the present study, PTRF mutations may not be rare in CGL patients.

This entity of generalized lipodystrophy with muscular dystrophy - which we believe to be novel - seems to represent a complicated disorder, as the occurrence of other symptoms could not readily be explained. Collection of detailed clinical information would therefore be essential in order to understand the precise function of PTRF.

\section{Methods}

Clinical materials. All clinical materials used in this study were obtained for diagnostic purposes and with informed consent. Subjects were selected from 2,745 muscular dystrophy specimens kept in the muscle repository of the National Center of Neurology and Psychiatry. The present studies were approved by the Ethical Committee at National Center of Neurology and Psychiatry.

Mutation screening and haplotype analysis. Genomic DNA was isolated from peripheral lymphocytes or muscles using standard techniques. All exons and their flanking intronic regions of PTRF, CAV3, LMNA, AGPAT2, BSCL2, CAV1, PPARG, AKT2, and ZMPSTE24 were directly sequenced using genomic DNA from all patients using an ABI PRISM 3100 automated sequencer (Applied Biosystems). Primer sequences are listed in Supplemental Table 3. To confirm the compound heterozygosity in P5, the PCR product was cloned and sequenced. In order to determine the frequency of the mutations in PTRF, we performed enzyme digestion of PCR products from 200 Japanese control subjects using Hpy188III (New England Biolabs) for c.696_697insC and TaqI (New England Biolabs) for c.525delG. MboII (New England Biolabs) was used for enzyme digestion of PCR products to detect the c.1138G>A substitution in BSCL2.

For haplotype analysis, we used 6 SNPs (rs2062213, rs8070945, rs963988, rs963987, rs963986, and rs9252) within PTRF. PCR products were analyzed by direct sequencing or enzyme digestion using MaeIII (Boehringer Mannheim). We also identified a novel 9-bp insertion polymorphism at the 


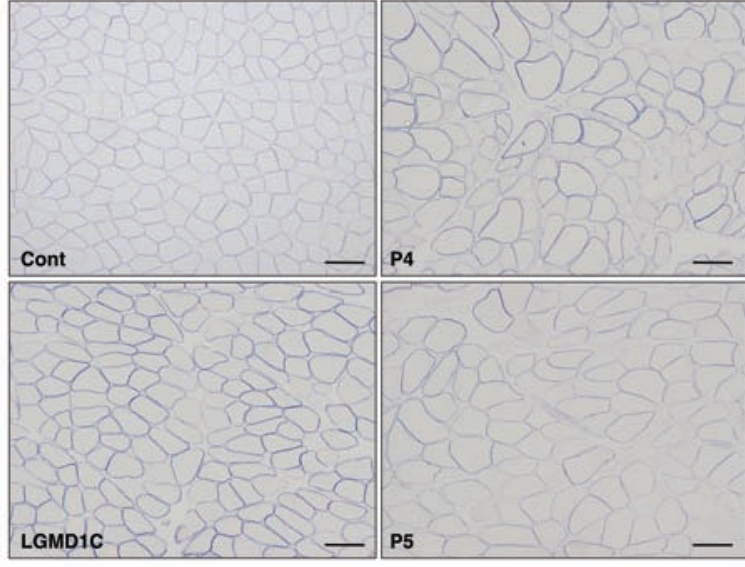

\section{Figure 8}

NDP activity assay. NDP activity was variable between muscle fibers, and was slightly increased in the muscle of P4, P5, and a LGMD1C patient with CAV3 mutation compared with an age-matched control subject. Scale bars: $100 \mu \mathrm{m}$.

$3^{\prime}$ noncoding region, and its frequency was calculated by PCR amplification using 50 Japanese control individuals. We also examined 2 microsatellite markers, STS-W93348 and D17S1185, the closest markers to PTRF. PCR product size was analyzed by GeneMapper using ABI 310 automated sequencer (Applied Biosystems).

Histochemical analysis. Biopsied muscle specimens were flash frozen with isopentane cooled in liquid nitrogen. Serial $10-\mu \mathrm{m}$-thick frozen sections were analyzed with 20 kinds of histochemical staining, including $\mathrm{H} \& \mathrm{E}$, modified Gomori trichrome, NADH-tetrazolium reductase, and oil red O. The NDP activity assay was performed to examine nNOS activity of each muscle fiber, as described previously (40). In brief, $10-\mu \mathrm{m}$-thick frozen sections were fixed with $4 \%$ paraformaldehyde in PBS for 2 hours at $4^{\circ} \mathrm{C}$. After a brief rinse with PBS, sections were incubated with $0.2 \%$ Triton X-100 in PBS for 20 minutes at $37^{\circ} \mathrm{C}$. The reaction was performed for 1 hour in a dark, humidified chamber at $37^{\circ} \mathrm{C}$ in $0.2 \%$ Triton X-100, $0.2 \mathrm{mM} \mathrm{NADPH}$, and $0.16 \mathrm{mg} / \mathrm{ml}$ nitro blue tetrazolium. The reaction was terminated by washing with water. We examined 6 age-matched controls and 2 LGMD1C patients with CAV3 mutations (p.R27G and p.E33K).

Immunohistochemical analysis. Immunostaining was performed using standard methods. Serial $6-\mu \mathrm{m}$-thick frozen muscle sections were fixed in cold acetone for 5 minutes. After blocking with normal goat serum, sections were incubated with the primary antibodies for 2 hours at $37^{\circ} \mathrm{C}$. We used antibodies against PTRF (A301-269A and A301-271A; BETHYL Laboratories), caveolin-1 (BD Biosciences), caveolin-2 (Sigma-Aldrich), caveolin-3 (BD Biosciences), and nNOS (BD Biosciences). Rabbit anti-PTRF antibody of A301-269A recognizes residue from 125 to 175 , and A301-271A was raised against residue 238 and 288 of human PTRF (Figure 1B). In order to exclude other diagnosable muscular dystrophies, we used antibodies for dystrophin (DYS1, DYS2, and DYS3; Novocastra); $\alpha$-, $\beta$-, $\gamma$-, and $\delta$-sarcoglycans (Novocastra); $\alpha$-dystroglycan (Upstate Biotech); $\beta$-dystroglycan (Novocastra); dysferlin (Novocastra); emerin (Novocastra); merosin (Chemicon); and collagen VI (ICN Biomedicals). After 6 rinses with PBS, sections were incubated with secondary antibodies of Alexa Fluor 488- or Alexa Fluor 568-labeled goat anti-mouse or -rabbit antibodies at room temperature for 45 minutes.

Immunoblotting analysis. Immunoblotting analysis was performed according to standard methods. Frozen muscle specimens were homogenized in SDS sample buffer and centrifuged at $15,000 \mathrm{~g}$ for 5 minutes. Protein $(20 \mu \mathrm{g})$ from each sample was loaded on $12 \%$ SDS-polyacrylamide gels and transferred to
PVDF membranes (Millipore). The membranes were blocked with 5\% skim milk in PBS and immunoreacted with antibodies to PTRF (A301-269A and A301-271A), caveolin-2, caveolin-3, nNOS, Smad2/3 (Cell Signaling Technology), p-Smad2/3 $3^{S 423 / 425}$ (Santa Cruz Biotechnology Inc.), Akt (Cell Signaling Technology), $\mathrm{p}-\mathrm{Akt}^{\mathrm{T} 308}$ (Cell Signaling Technology), and $\mathrm{p}-\mathrm{Akt}^{\mathrm{S} 473}$ (Cell Signaling Technology) overnight at $4^{\circ} \mathrm{C}$. After washing in PBS containing $0.1 \%$ Tween-20, the membrane was incubated with horseradish peroxidaselabeled secondary antibody and visualized with ECL (Amersham Pharmacia Biotech). Data were analyzed using LAS-1000 chemiluminescence imaging system (Fujifilm). Quantification of immunoreactive bands was performed by densitometric analysis using Quantity One (PDI), and protein amounts for caveolin-3 and nNOS were normalized by the intensity of MHC. The ratio of p-Smad $2 / 3$ and p-Akt, to Smad and Akt, respectively, was also calculated.

Electron microscopy. Muscle specimens were fixed with $2 \%$ glutaraldehyde in $0.1 \mathrm{M}$ cacodylate buffer. After shaking with a mixture of $4 \%$ osmium tetroxide, $1.5 \%$ lanthanum nitrate, and $0.2 \mathrm{M}$ s-collidine for 2-3 hours, samples were embedded in epoxy resin. Semithin sections ( $1 \mu \mathrm{m}$ thick) were stained with toluidine blue. Ultrathin sections $50 \mathrm{~nm}$ thick were stained with uranyl acetate and lead citrate, then examined under H-600 transmission electron microscope (Hitachi) at $75 \mathrm{kV}$.

$R T-P C R$. Total RNA was extracted from biopsied skeletal muscles using TRIzOL (Invitrogen), and RT-PCR was performed using SuperScript III (Invitrogen) with random hexamer according to the manufacturer's instructions. Primers for each gene were located on different exons or directly spanning exon-exon boundaries of the genomic sequence in order to minimize amplification from any contaminating genomic DNA. After performing preliminary gradient PCR assays, the optimal annealing temperature for all the primer pairs was determined in order to generate the lowest $\mathrm{Ct}$ value as well as a sharp melting peak, with no amplification of nonspecific products or primer-dimer artifacts. Quantitative RT-PCR was performed to compare the mRNA expression of caveoin-1, caveolin-2, caveolin-3, and myostatin using Rotor-Gene 6000 according to the manufacturer's instructions (Corbett Life Science). The reactions were performed in reference to the GAPDH. We used 4 points consisting of 10 -fold serial dilution using each primer set to build the standard curve. The PCR reaction (50 cycles) was followed by a melting curve analysis, ranging from $72^{\circ} \mathrm{C}$ to $95^{\circ} \mathrm{C}$, with temperature increasing steps of $0.5^{\circ} \mathrm{C}$ every 10 seconds. Baseline and threshold values were automatically determined and analyzed. $R^{2}$ values exceeded 0.97 . The 2 -standard curve method was used to determine the relative expression ratio of the target gene in the patient samples versus the control sample, with reference to GAPDH expression.

Cell culture and transfection. COS-7 and C2C12 cells were maintained at $37^{\circ} \mathrm{C}$ in a humidified atmosphere of $5 \% \mathrm{CO}_{2}$ in DMEM (Sigma-Aldrich) supplemented with $10 \%$ fetal bovine serum. Full-length PTRF and caveolin-1 and -3 were amplified using total RNA from control human muscle and cloned into the pGEM-T-easy vector (Promega). The PTRF mutants c.525delG and c.696_697ins $C$ were generated using appropriate primers. All primer sequences are shown in Supplemental Table 3.

Immunocytochemistry. COS-7 and C2C12 myoblasts were cotransfected with FLAG-tagged WT or mutant (c.525delG or c.696_697insC) PTRF cDNA and T7-tagged human caveolin-3 using FuGENE6 (Roche). Transfectants were fixed for 30 minutes in $2 \%$ paraformaldehyde or $100 \%$ methanol, then permeabilized in $0.1 \%$ Triton X-100 for 10 minutes. Polyclonal antibodies to FLAG (Sigma-Aldrich) with caveolin-3 (BD Biosciences) or FLAG with $\beta$-tubulin (Calbiochem) were applied for double staining.

Immunoprecipitation. COS-7 cells were cotransfected with FLAG-tagged WT or mutant (c.525delG or c.696_697insC) PTRF cDNA and T7-tagged human caveolin-1 or caveolin-3 using FuGENE6 (Roche). The sequences of all constructs were verified with DNA sequencing using ABI PRISM 310 (Applied Biosystems). After 48 hours, the lysates from transfectants were 
solubilized with $50 \mathrm{mM}$ Tris- $\mathrm{HCl}$ (pH 7.5), $150 \mathrm{mM} \mathrm{NaCl}, 50 \mathrm{mM}$ EDTA, $1 \%$ Triton X-100, and Complete-mini EDTA-free proteinase inhibitors (Roche) (9). The solubilized lysate precleared with Protein G Sepharose (GE Healthcare) was incubated with anti-FLAG (M2; Sigma-Aldrich) and anti-T7 (Novagen) antibodies. Immunoprecipitated proteins were dissociated from beads by boiling in sample buffer and were resolved by SDSPAGE. Immunoblotting was performed using standard techniques.

\section{Acknowledgments}

We thank Sherine Shalaby (Heliopolis Neurocenter, Cairo, Egypt) and May Malicdan (National Center of Neurology and Psychiatry) for reviewing the manuscript. This study was supported by "Research on Psychiatric and Neurological Diseases and Mental Health" of "Health Labour Sciences Research Grant" and by "Research Grant (20B-12, 20B-13) for Nervous and Mental Disorders" from the Ministry of Health, Labor, and Welfare of Japan; by grants from the Human Frontier Science Program; by a Grant-in-Aid for Scientific Research from Japan Society for the Promotion of Science; by Research on Publicly Essential Drugs and Medical Devices from the Japanese Health Sciences Foundation; and by the Program for Promotion of Fundamental Studies in Health Sciences of the National Institute of Biomedical Innovation (NIBIO).

Received for publication January 20, 2009, and accepted in revised form June 3, 2009.

Address correspondence to: Yukiko K. Hayashi, Department of Neuromuscular Research, National Institute of Neuroscience, National Center of Neurology and Psychiatry, 4-1-1 Ogawahigashi, Kodaira, 187-8502 Tokyo, Japan. Phone: 81-42-341-2711, Fax: 81-42-346-1742; E-mail: hayasi_y@ncnp.go.jp.
1. Scherer, P.E., et al. 1997. Cell-type and tissue-specific expression of caveolin-2. Caveolins 1 and 2 co-localize and form a stable hetero-oligomeric complex in vivo. J. Biol. Chem. 272:29337-29346.

2. Tang, Z., et al. 1996. Molecular cloning of caveolin-3, a novel member of the caveolin gene family expressed predominantly in muscle. J. Biol. Chem. 271:2255-2261.

3. Galbiati, F., Razani, B., and Lisanti, M.P. 2001. Emerging themes in lipid rafts and caveolae. Cell. 106:403-411.

4. Thomas, C.M., and Smart, E.J. 2008. Caveolae structure and function. J. Cell. Mol. Med. 12:796-809.

5. Hill, M.M., et al. 2008. PTRF-Cavin, a conserved cytoplasmic protein required for caveola formation and function. Cell. 132:113-124.

6. Liu, L., et al. 2008. Deletion of Cavin/PTRF causes global loss of caveolae, dyslipidemia, and glucose intolerance. Cell Metab. 8:310-317.

7. Minetti, C., et al. 1998. Mutations in the caveolin-3 gene cause autosomal dominant limb-girdle muscular dystrophy. Nat. Genet. 18:365-368.

8. Matsuda, C., et al. 2001. The sarcolemmal proteins dysferlin and caveolin-3 interact in skeletal muscle. Hum. Mol. Genet. 10:1761-1766.

9. Liu, L., and Pilch, P.F. 2008. A critical role of cavin (polymerase I and transcript release factor) in caveolae formation and organization. J. Biol. Chem. 283:4314-4322.

10. Minetti, C., et al. 2002. Impairment of caveolae formation and T-system disorganization in human muscular dystrophy with caveolin-3 deficiency. Am. J. Pathol. 160:265-270.

11. Ohsawa, Y., et al. 2006. Muscular atrophy of caveolin-3-deficient mice is rescued by myostatin inhibition. J. Clin. Invest. 116:2924-2934.

12. Frost, R.A., and Lang, C.H. 2007. Protein kinase B/Akt: a nexus of growth factor and cytokine signaling in determining muscle mass. J. Appl. Physiol. 103:378-387.

13. Venema, V.J., Ju, H., Zou, R., and Venema, R.C. 1997. Interaction of neuronal nitric-oxide synthase with caveolin-3 in skeletal muscle. Identification of a novel caveolin scaffolding/inhibitory domain. J. Biol. Chem. 272:28187-28190.

14. Sunada, Y., et al. 2001. Transgenic mice expressing mutant caveolin-3 show severe myopathy associated with increased nNOS activity. Hum. Mol. Genet. 10:173-178.
15. Agarwal, A.K., et al. 2002. AGPAT2 is mutated in congenital generalized lipodystrophy linked to chromosome 9q34. Nat. Genet. 31:21-23.

16. Magre, J., et al. 2001. Identification of the gene altered in Berardinelli-Seip congenital lipodystrophy on chromosome 11q13. Nat. Genet. 28:365-370.

17. Kim, C.A., et al. 2008. Association of a homozygous nonsense caveolin-1 mutation with BerardinelliSeip congenital lipodystrophy. J. Clin. Endocrinol. Metab. 93:1129-1134.

18. Cao, H., Alston, L., Ruschman, J., and Hegele, R.A. 2008. Heterozygous CAV1 frameshift mutations (MIM 601047) in patients with atypical partial lipodystrophy and hypertriglyceridemia. Lipids Health Dis. 7:3.

19. Cao, H., and Hegele, R.A. 2000. Nuclear lamin A/C R482Q mutation in canadian kindreds with Dunnigan-type familial partial lipodystrophy. Hum. Mol. Genet. 9:109-112.

20. Agarwal, A.K., et al. 2003. Phenotypic and genetic heterogeneity in congenital generalized lipodystrophy. J. Clin. Endocrinol. Metab. 88:4840-4847.

21. George, S., et al. 2004. A family with severe insulin resistance and diabetes due to a mutation in AKT2. Science. 304:1325-1328.

22. Barroso, I., et al. 1999. Dominant negative mutations in human PPARgamma associated with severe insulin resistance, diabetes mellitus and hypertension. Nature. 402:880-883.

23. Hegele, R.A., et al. 2006. Sequencing of the reannotated LMNB2 gene reveals novel mutations in patients with acquired partial lipodystrophy. Am. J. Hum. Genet. 79:383-389.

24. Aboulaich, N., Ortegren, U., Vener, A.V., and Stralfors, P. 2006. Association and insulin regulated translocation of hormone-sensitive lipase with PTRF. Biochem. Biophys. Res. Commun. 350:657-661.

25. Razani, B., et al. 2002. Caveolin-1-deficient mice are lean, resistant to diet-induced obesity, and show hypertriglyceridemia with adipocyte abnormalities. J. Biol. Chem. 277:8635-8647.

26. Fulizio, L., et al. 2005. Molecular and muscle pathology in a series of caveolinopathy patients. Hum. Mutat. 25:82-89.

27. Betz, R.C., et al. 2001. Mutations in CAV3 cause mechanical hyperirritability of skeletal muscle in rippling muscle disease. Nat. Genet. 28:218-219.

28. Sugie, K., et al. 2004. Two novel CAV3 gene muta- tions in Japanese families. Neuromuscul. Disord. 14:810-814.

29. Hayashi, T., et al. 2004. Identification and functional analysis of a caveolin-3 mutation associated with familial hypertrophic cardiomyopathy. Biochem. Biophys. Res. Commun. 313:178-184.

30. Vatta, M., et al. 2006. Mutant caveolin-3 induces persistent late sodium current and is associated with long-QT syndrome. Circulation. 114:2104-2112.

31. Park, D.S., et al. 2002. Caveolin-1/3 double-knockout mice are viable, but lack both muscle and nonmuscle caveolae, and develop a severe cardiomyopathic phenotype. Am. J. Pathol. 160:2207-2217.

32. Woodman, S.E., et al. 2002. Caveolin-3 knock-out mice develop a progressive cardiomyopathy and show hyperactivation of the $\mathrm{p} 42 / 44$ MAPK cascade. J. Biol. Chem. 277:38988-38997.

33. Zhao, Y.Y., et al. 2002. Defects in caveolin-1 cause dilated cardiomyopathy and pulmonary hypertension in knockout mice. Proc. Natl. Acad. Sci. U. S. A. 99:11375-11380

34. Xu, Y., Buikema, H., van Gilst, W.H., and Henning, R.H. 2008. Caveolae and endothelial dysfunction: filling the caves in cardiovascular disease. Eur. J. Pharmacol. 585:256-260.

35. El-Yazbi, A.F., Cho, W.J., Boddy, G., and Daniel, E.E. 2005. Caveolin-1 gene knockout impairs nitrergic function in mouse small intestine. Br. J. Pharmacol. 145:1017-1026.

36. Lobie, P.E., Sadir, R., Graichen, R., Mertani, H.C., and Morel, G. 1999. Caveolar internalization of growth hormone. Exp. Cell Res. 246:47-55.

37. Rajab, A., Heathcote, K., Joshi, S., Jeffery, S., and Patton, M. 2002. Heterogeneity for congenital generalized lipodystrophy in seventeen patients from Oman. Am. J. Med. Genet. 110:219-225.

38. Ghanem, Q. 1993. Percussion myoedema in a Pakistani boy with Berardinelli Seip lipodystrophy syndrome. Clin. Genet. 44:277-278.

39. Simha, V., Agarwal, A.K., Aronin, P.A., Iannaccone, S.T., and Garg, A. 2008. Novel subtype of congenital generalized lipodystrophy associated with muscular weakness and cervical spine instability. Am J. Med. Genet. A. 146A:2318-2326.

40. Kameya, S., et al. 1999. alpha1-syntrophin gene disruption results in the absence of neuronal-type nitric-oxide synthase at the sarcolemma but does not induce muscle degeneration. J. Biol. Chem. 274:2193-2200. 